\title{
Editorial
}

\section{ENSEÑANZA EN ENFERMERÍA EDUCACIÓN, CAPACITACIÓN Y DESARROLLO}

El proceso educativo es continuo, dinámico y se puede llevar a cabo en diferentes tiempos y espacios. De acuerdo a la Constitución Política de los Estados Unidos Mexicanos'? las empresas estarán obligadas a proporcionar capacitación o adiestramiento para el trabajo a sus empleados, mismo que se ve reflejado en la Ley Federal del trabajo, Articulo 153 que se refiere a la Capacitación y Adiestramiento para el trabajo?

En el Instituto Nacional de Neurología y Neurocirugía, Manuel Velasco Suárez (INNN MVS) la Coordinación de Enseñanza en Enfermería depende jerárquicamente de la Subdirección de enfermería, por lo cual asume un papel muy importante en la educación continua, capacitación y desarrollo del personal de adscrito a esta subdirección. Sustenta su actuar a razón de la misión y visión institucional las cuales van dirigidas a la formación de recursos humanos que permita:

Brindar atención médica de excelencia a pacientes con padecimientos y/o afecciones del sistema nervioso, y contribuir a la estandarización nacional del manejo de pacientes con trastornos neurológicos y psiquiátricos.

Para cumplir con esta tarea es necesario definir tres términos que orientan el rumbo de la enseñanza:

1. Capacitación: es un proceso activo y permanente que consiste en adquirir, mantener, renovar, reforzar, actualizar e incrementar conocimientos, destrezas y actitudes para mejorar la competencia del personal que ha terminado su instrucción formal ${ }^{3}$ y que le permitan enfrentar los cambios tecnológicos y de innovación productiva de manera competente y provechosa.

En relación a este apartado se llevan a cabo:
- Diplomados dirigidos a el área quirúrgica, de investigación y cuidado crítico, entre otros.

- Residencia en enfermería (práctica especializada), se lleva a cabo por egresados de las especialidades en enfermería de la ENEO-UNAM, como una opción de titulación y una forma de obtener más habilidad en la práctica especializada. Es relevante mencionar que, a partir del año 2008, dio inicio la residencia de enfermería al adulto en estado crítico, actualmente contamos con residentes de las especialidades de Enfermería Neurológica, del Adulto en Estado Crítico, Salud Mental, Salud Pública y Enfermería Perioperatorio.

Estancia clínico- académica. Se han realizado en dos direcciones:

a) El personal adscrito a nuestra institución, acude a los diferentes institutos nacionales, como son el Instituto Nacional de Rehabilitación, Instituto Nacional de Cardiología e Instituto Nacional de Ciencias Médicas y Nutrición, entre otros, con la finalidad de que el personal adquiriera habilidades en áreas de especialidad que conformen su competencia profesional, como son el cuidado de la persona en áreas como la rehabilitación neurológica, el manejo de personas con tratamientos nefrológicos como diálisis y hemodiálisis, y aspectos de enfermería cardiovascular.

b) Por otra parte en el instituto se han recibido enfermeras, que han venido a las áreas de cuidado crítico y quirúrgico, de instituciones como Hospital Regional de Alta Especialidad Ciudad Salud de Tapachula Chiapas, Hospital General de México, Hospital Regional de Alta Especialidad de Oaxaca y de Veracruz.

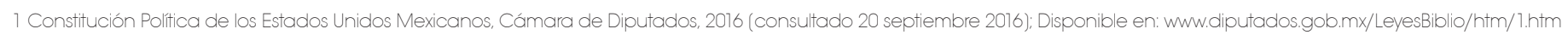
2 Ley Federal del Trabajo, Secretaría de Trabajo y Previsión Social, 2016 (consultado 20 septiembre 2016); Disponible en: info4.juridicas.unam.mx/juslab/leylab/123/156.htm

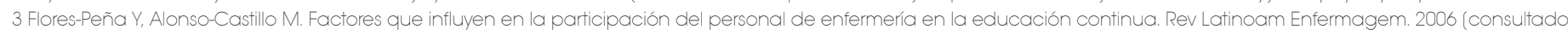
20 septiembre 2016); 14(3): (8 p.). Disponible en: www.scielo.br/pdf/rlae/v14n3/es_v14n3a02.pdf

Enf Neurol Vol. 15. No. 3 septiembre - diciembre 2016 
2. Educación continua: el proceso de formación y/o actualización en conocimientos y habilidades al que las personas se someten con el propósito de modificar actitudes, conductas y hábitos, mejorar el desempeño profesional y erradicar rutinas.

La programación de los cursos de capacitación continua se realiza considerando los siguientes aspectos:

Necesidades detectadas y manifestadas del personal Epidemiología

Programas prioritarios

Problemas de salud emergentes

Necesidades del servicio

Innovación y cambios tecnológicos

Educación para la salud

3. Desarrollo: Consiste en mejorar habilidades e incrementar conocimientos en áreas específicas, cambiar de actitudes para generar personas proactivas

A partir del año 2007, la Coordinación de Enseñanza en Enfermería y el Departamento de Capacitación y Enseñanza en Enfermería, tienen como función principal ejecutar estas tres acciones en forma constante, dirigido tanto al personal de enfermería, como al personal de áreas afines, con el objetivo de brindar una mejor calidad de atención a nuestros usuarios desde cualquier área donde se desempeñen.

Se coadyuva en la formación profesional de los estudiantes de enfermería de pregrado, posgrado y pasantes de enfermería; a través de la coordinación y convenios con las instituciones educativas y de salud que solicitan las instalaciones del instituto, como la Escuela Nacional de Enfermería y Obstetricia de la Universidad Nacional Autónoma de México, Escuela Superior de Enfermería y Obstetricia del Instituto Politécnico Nacional (IPN), Centro Interdisciplinario de Ciencias de la Salud del IPN, Instituto Marillac, Facultad de Estudios Superiores Iztacala, Universidad Panamericana, Universidad Autónoma de San Luis Potosí, Universidad Autónoma del Estado de Morelos, Universidad Autónoma de Tlaxcala.

En 2008 y 2009 continuábamos siendo sede del Postécnico de Enfermería Psiquiátrica.

Desde el año 2005 somos sede de la Especialidad en Enfermería Neurológica, y de la Especialidad en Enfermería del Adulto en Estado Crítico a partir de 2012.
Se apoyó al personal de enfermería para obtener la licenciatura en enfermería en años posteriores, a través del Sistema de Universidad Abierta en línea, en la ENEOUNAM en el año 2009 y 2010.

\section{Avances de la Coordinación de Enseñanza en Enfermería son:}

La coordinación de educación lleva a cabo diversas actividades académicas entre ellas están la organización de la Jornada de Enfermería la cuál tiene diferente temática cada año, a partir de las necesidades nacionales como institucionales en materia de educación para la salud y actualización.

La jornada propiamente es un foro de intercambio académico en donde los conocimientos en la atención del enfermo neurológico, se actualizan y se vinculan con los avances teóricos, metodológicos procedimentales de vanguardia.

Se ha trabajado para contar docentes nacionales como extranjeros de instituciones de salud y educativas que nos dan a conocer los avances científicos, tecnológicos y de atención que existen para el cuidado del enfermo con riesgo y daño neurológico, neuropsiquiatricas y neuroquirúrgico. En 2016 al celebrar el XXX aniversario de este importante evento científico de enfermería el tema central de la Jornada fue "Mirando hacia el futuro", y en él se trataron temas relacionados con las reformas en materia de salud, así como la influencia de las tecnologías de la información en enfermería, como también los nuevos tratamientos en el enfermo con enfermedad de Parkinson, entre otros.

Dentro del aspecto administrativo esta coordinación en conjunto con la subdirección en enfermería propiamente, elaboró la hoja de registros clínicos de enfermería que actualmente se utiliza, así como el pilotaje, capacitación, e implementación en el año 2009.

Es relevante mencionar que en el año 2009 se obtuvo la Certificación en Enfermería y recertificación en 2015, por lo cual la labor en este rubro es continua, para ello uno de los puntos a mejorar es la certificación del profesional enfermero es a bien comentar que en el mes de septiembre de 2016, el instituto cuenta con 26 enfermeras certificadas, de están cinco se recertificaron, es contundente el grado académico del enfermero mismo que se muestra en las gráficas siguientes:

\section{6 MMN/Why Enf Neurol Vol. 15. No. 3 septiembre - diciembre 2016}


Gráfica 1. Grado académico de enfermeras certificadas a septiembre de 2015

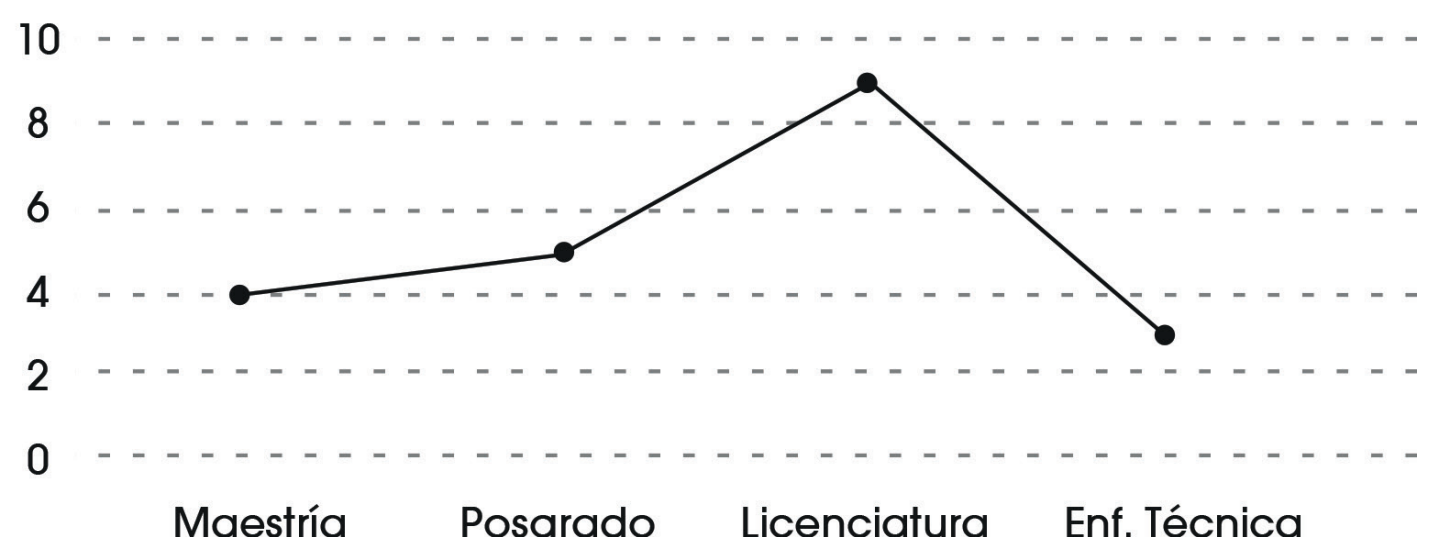

Gráfica 2. Grado académico de enfermeras recertificadas a septiembre de 2015

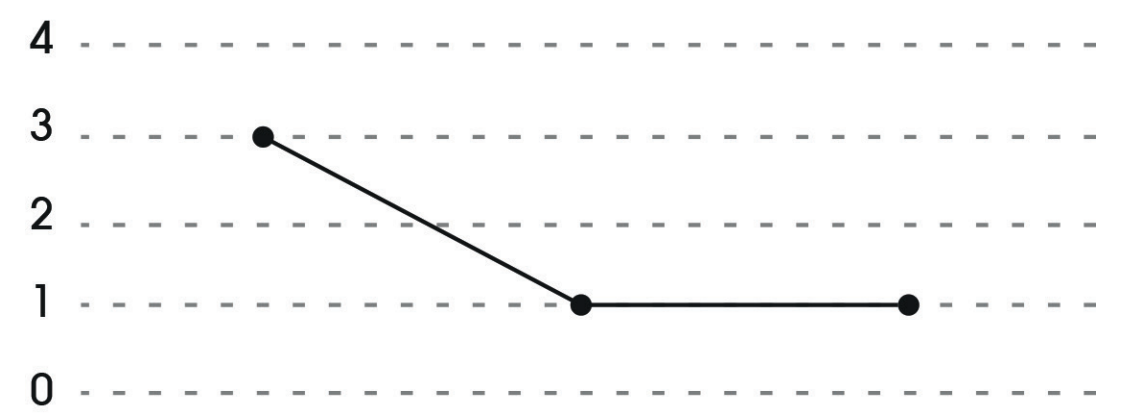

Maestría Posgrado Licenciatura

Fuente: Archivos del Departamento de Capacitación y Enseñanza en Enfermería, 2015

Además, el responsable de esta área ha participado activamente en el proceso de corte, es decir en el examen de certificación y elaboración de reactivos, para determinar niveles de desempeño, en el intervalo de 2009 a 2016. Del Centro Nacional de Evaluación para la Educación Superior (Ceneval)

Al formar parte del Comité de calidad desde el año 2011, se ha trabajado en la coordinación y desarrollo de la Guía de Práctica Clínica de Enfermería (GPCE):

Intervenciones de enfermería en la atención de las personas con secuelas neurológicas en el segundo y tercer nivel, 2014.

Esto último en colaboración con personal del Instituto Nacional de Rehabilitación, Instituto Nacional de Ciencias Médicas y Nutrición. 
Rev. Enf. Neurol. (Mex)

Desarrollando actualmente también la GPCE:

Intervención de enfermería para la detección oportuna y control del Episodio depresivo mayor sin síntomas psicóticos en mujeres mayores de 18 años en los tres niveles de atención,

En colaboración con Servicios de Atención Psiquiátrica, Instituto de Psiquiatría Ramón de la Fuente e Instituto Nacional de Ciencias Médicas y Nutrición. Trabajos coordinados por la Dirección General de Calidad y Educación en salud, de la Secretaría de Salud y el Centro Nacional de Excelencia Tecnológica en Salud (CENETEC)

Sin restar importancia se participa activamente en el proyecto de Consultoría de Enfermería Neurológica el cual fue galardonado en el año 2014.
Entre todos los rubros descritos, uno de los aspectos a resaltar es la implementación de programas de capacitación, como método para desarrollar las competencias profesionales del personal de enfermería donde el impacto se verá reflejado en un primer momento en la calidad del cuidado especializado, en la organización y gestión del mismo, propiciando un ambiente y clima laboral, en donde aspectos de seguridad en el cuidado se vean favorecidos, así mismo la satisfacción del profesional al compartir sus conocimientos con profesionales en formación, incentiva el uso e innovación de nuevas tecnologías, lo que construye la profesionalización en enfermería.

MASS. Silvia Alejandro Escobar Coordinadora de Enseñanza en Enfermería

\section{8} MMN WMy, Enf Neurol Vol. 15. No. 3 septiembre - diciembre 2016 